\title{
Tunable 3D nanofibrous and bio-functionalised PEDOT network explored as a conducting
} polymer-based biosensor.

\author{
Lingyin Meng, Anthony P. F. Turner ${ }^{+}$, and Wing Cheung Mak* \\ Biosensors and Bioelectronics Centre, Division of Sensor and Actuator Systems, Department of Physics, Chemistry and \\ Biology, Linköping University, SE-581 83 Linköping, Sweden \\ ${ }^{+}$Current address: Professor Emeritus, SATM, Cranfield University, Bedfordshire, MK430AL, UK. \\ *Corresponding author. \\ E-mail address: wing.cheung.mak@liu.se (W.C. Mak).
}

\begin{abstract}
Conducting polymers that possess good electrochemical properties, nanostructured morphology and functionality for bioconjugation are essential to realise the concept of all-polymer-based biosensors that do not depend on traditional nanocatalysts such as carbon materials, metal, metal oxides or dyes. In this research, we demonstrated a facile approach for the simultaneous preparation of a bi-functional PEDOT interface with a tunable 3D nanofibrous network and carboxylic acid groups (i.e. Nano-PEDOT-COOH) via controlled co-polymerisation of EDOT and EDOT-COOH monomers, using tetrabutylammonium perchlorate as a soft-template. By tuning the ratio between EDOT and EDOT-COOH monomer, the nanofibrous structure and carboxylic acid functionalisation of Nano-PEDOT-COOH were varied over a fibre diameter range of $15.6 \pm 3.7$ to $70.0 \pm 9.5 \mathrm{~nm}$ and a carboxylic acid group density from 0.03 to $0.18 \mu \mathrm{mol}$ $\mathrm{cm}^{-2}$. The nanofibres assembled into a three-dimensional network with a high specific surface area, which contributed to low charge transfer resistance and high transduction activity towards the co-enzyme NADH, delivering a wide linear range of 20-960 $\mu \mathrm{M}$ and a high sensitivity of $0.224 \mu \mathrm{A} \mu \mathrm{M}^{-1} \mathrm{~cm}^{-2}$ at the Nano-PEDOT-COOH50\% interface. Furthermore, the carboxylic acid groups provide an anchoring site for the stable immobilisation of an NADH-dependent dehydrogenase (i.e. lactate dehydrogenase), via EDC/S-NHS chemistry, for the fabrication of a Bio-Nano-PEDOT-based biosensor for lactate detection which had a response time of less than $10 \mathrm{~s}$ over the range of $0.05-1.8 \mathrm{mM}$. Our developed bio-Nano-PEDOT interface shows future
\end{abstract}


potential for coupling with multi-biorecognition molecules via carboxylic acid groups for the development of a range of advanced all-polymer biosensors.

Keywords: conducting polymer, bio-PEDOT, nanofibre, carboxylic acid functionalisation, biosensor interface

\section{Highlights}

(1) 3D Nano-PEDOT-COOH network possessing a nanofibrous structure and carboxylic acid groups were realised simultaneously via facile soft-template electropolymerisation.

(2) The nanofibrous structure was favourable for probe charge transfer and NADH transduction.

(3) The high density of carboxylic acid groups provided anchoring sites for stable immobilisation of an NADH-dependent dehydrogenase to create Bio-Nano-PEDOT interface.

(4) The Bio-Nano-PEDOT interface showed good analytical performance for NADH sensing and lactate biosensing.

\section{Introduction}

Poly(3,4-ethylenedioxythiophene) (PEDOT), an emerging conducting polymer (CP) with extraordinary redox reversibility, biocompatibility and electrochemical properties, shows considerable potential as an interfacing material in biosensing to bridge between the organic electronics world and bioelectronics, since PEDOT possess sufficient similarities in its organic chemical nature with biological molecules to facilitate the concept of all-polymer biosensors (Inal et al. 2018). One key to merge organic electronics and bioelectronics is to establish an effective signal transduction interface between biomolecules and PEDOT. However, the lack of reactive functional groups for bioconjugation and the relatively low electrochemical catalytic activity of pristine PEDOT, has hindered the development of a direct PEDOT-based electrochemical biosensors with bioelectrochemical signal transduction between the catalytic 
enzyme and PEDOT. In most of the reported PEDOT sensors and biosensors, nanomaterials were used as dopants to prepare PEDOT-composites, including carbon materials (carbon nanotubes, graphene), metal or metal oxides $\left(\mathrm{Au}, \mathrm{TiO}_{2}\right)$ and dyes (Prussian blue) (Lin et al. 2010; Meng et al. 2019b; Wang et al. 2018). The PEDOT mainly served as a conducting matrix while the bioelectrochemical signal transduction still relied on the doped carbon, metal or dye nanocatalyst. Thus, pure PEDOT interfaces possessing functionality for both bioconjugation and good electrochemical activity are still largely absent while remaining critical for future advances in biosensing.

Functionalisation endows conducting polymers (CPs) with functional groups that facilitate effective immobilisation of biorecognition molecules for biosensing applications. In recent years, much effort has been devoted to design functionalised CPs with active chemical groups, such as carboxylic acid (Luo et al. 2009), amine (Godeau et al. 2016), phosphorylcholine (Goda et al. 2015), sialic acid (Hai et al. 2017) etc.. Of these, the functionalisation of CPs with carboxylic acid groups delivers the most value compared to the others because of the variety of biological molecules, such as nucleotides and proteins (antibodies and enzymes), that can be covalently immobilised on the $\mathrm{CP}$ transducer interface, via ethyl(dimethylaminopropyl) carbodiimide (EDC)/N-hydroxysulfosuccinimide (NHS) chemistry. The introduction of carboxylic acid groups can be achieved either by using carboxylic acid containing molecules as a dopant during polymerisation or by using their derivatives (e.g. EDOT-COOH) as monomers. Carboxylic acid containing molecules, such as citrate (Meng et al. 2019a) and hyaluronic acid (Mantione et al. 2016) can be incorporated into the CP matrix as a dopant to compensate for the backbone charge, thus endowing the resulting $\mathrm{CP}$ interface with a high - $\mathrm{COOH}$ density. However, this strategy suffers from several drawbacks including potential leakage of dopant, difficulty in controlling the amount of dopant and destruction of the CP's bulk properties and conductivity. Alternatively, CP derivatives bearing carboxylic acid groups have been used for the preparation of a close-structured planar functionalised PEPOT film coupled with DNA and or antibodies for indirect affinity biosensing (Luo et al. 2009; Sekine et al. 2011).

Advances in nanomaterials based on conventional carbon (CNTs, graphene) and metal 
nanomaterials ( $\mathrm{Au}, \mathrm{Pt}$ nanoparticles) used in bioelectronics (Jin et al. 2018a; Jin et al. 2018b) encourages the exploration of nanostructured CPs. Nanostructured CPs inherit prominent physical-chemical properties from their bulk polymer equivalents, while acquiring beneficial nanomaterial characteristics such as large active surface area to promote catalytic reactions. While bulk CP interfaces may suffer from hysteresis and sluggish electron/mass transfer, nanostructured CPs are capable of realising effective electrode interface kinetics and facilitate signal transfer due to their high surface area and porous structure, leading to faster response times and high sensitivity for the resulting sensors (Lu et al. 2019). Various CP nanostructures, including nanospheres (Nguyen et al. 2015), nanowires (Travas-Sejdic et al. 2014) and nanotubes (Lin et al. 2016), have been implemented via sacrificial hard-template (e.g. polystyrene), soft-template (e.g. surfactants) or template-free (dimer as seeds) fabrication. Although these techniques yield a controlled nanostructured CP interface, the demand for a high density of functional groups for biorecognition elements still remains unfulfilled.

In this study, we developed an innovative bioorganic/organic polymer bio-interface possessing functionality for bioconjugation and good electrochemical properties for signal transduction towards an all-polymer-based biosensor. By combining functionalisation and nanostructure formation strategies, we demonstrate herein a facile approach for the simultaneous preparation of a bi-functional PEDOT interface with a tunable 3D nanofibrous network and carboxylic acid groups (i.e. Nano-PEDOT-COOH) via controlled copolymerisation of EDOT and EDOT-COOH monomers using tetrabutylammonium perchlorate as a soft-template (Scheme 1a). The ratio between EDOT and EDOT-COOH was evaluated to modulate the resulting nanostructure morphologies and density of the carboxylic acid groups. The 3D nanofibrous network endowed the Nano-PEDOT-COOH interface with enhanced charged transfer and catalytic activity towards the electrochemical oxidation of NADH, which may be ascribed to the relatively large 3D active surface area and porous structure. Furthermore, the carboxylic acid functionality provided anchoring sites for the stable immobilisation of a $\mathrm{NADH}$-dependent dehydrogenase (i.e. lactate dehydrogenase) via EDC/S-NHS chemistry for the fabrication of a Bio-Nano-PEDOT-based biosensor for lactate detection that delivers a fast 
response time and high sensitivity (Scheme 1b). This strategy also provides a generic design for electrocatalytic devices based on the many available NADH-dependent dehydrogenase enzymes.

\section{a Nano-PEDOT-COOH}

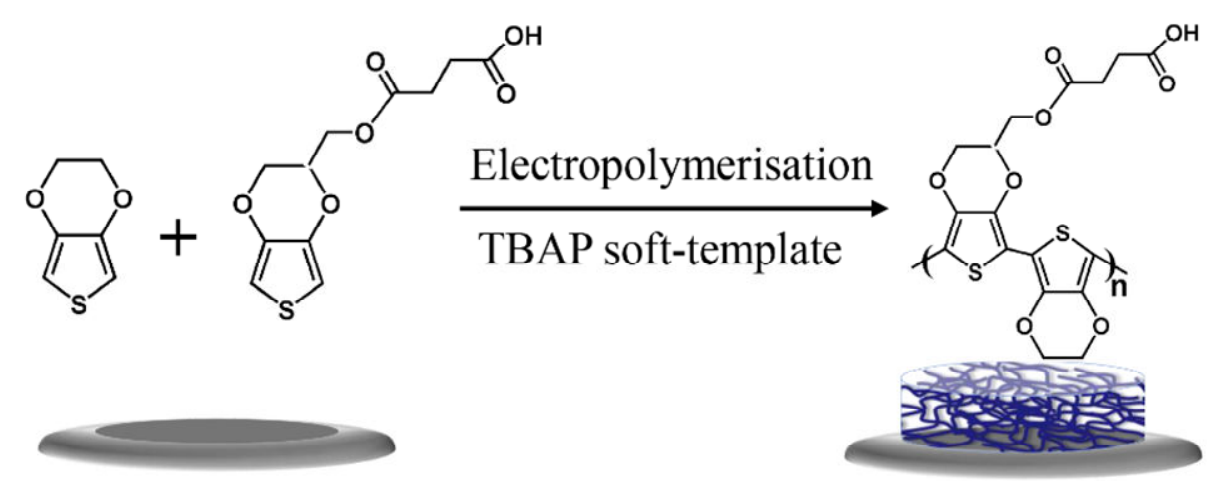

\section{b Bio-Nano-PEDOT}
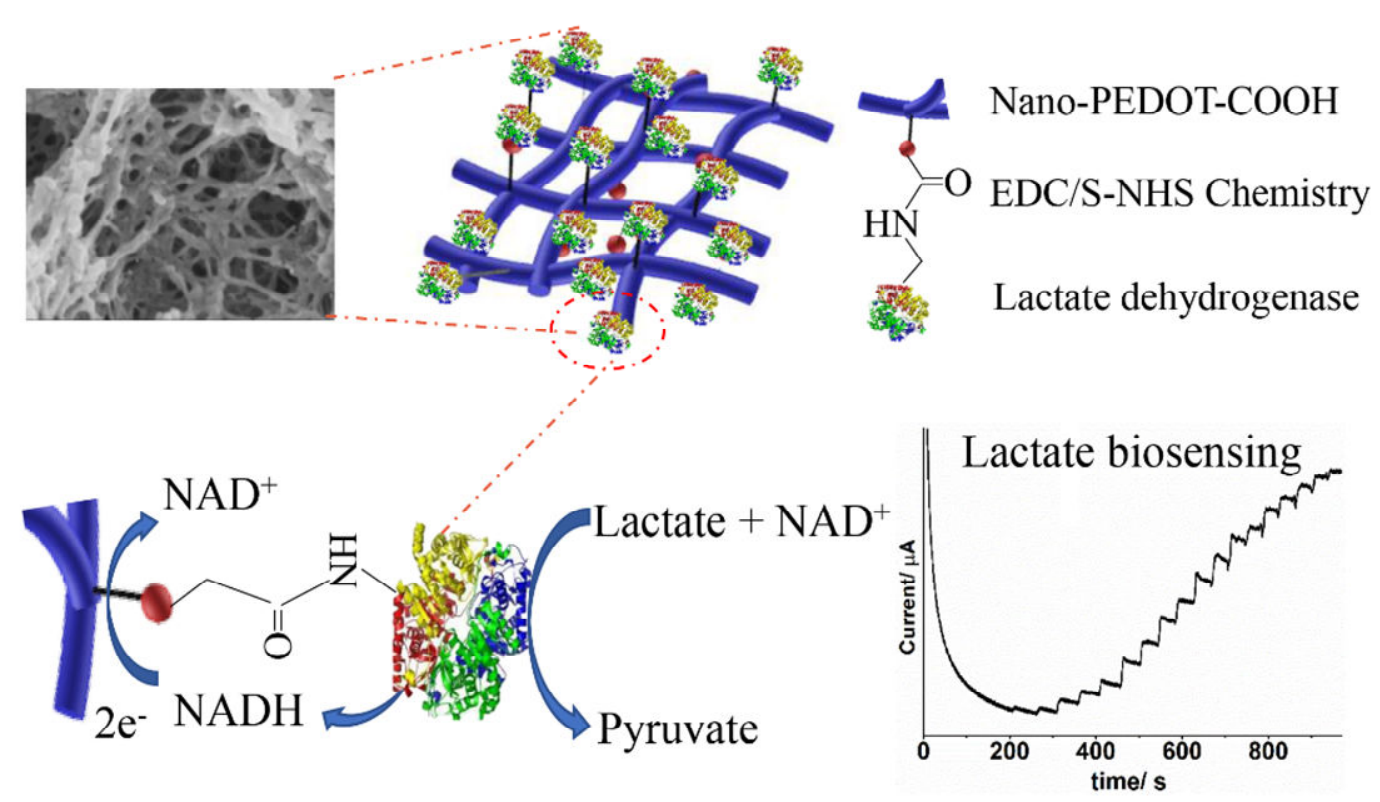

Scheme 1. Schematic of (a) 3D Nano-PEDOT-COOH network preparation via copolymerisation of EDOT and EDOT-COOH monomers using tetrabutylammonium perchlorate as a soft-template ; (b) Bio-Nano-PEDOT: Nano-PEDOT-COOH bio-conjugation with Lactate dehydrogenase via EDC/S-NHS chemistry for lactate biosensing.

\section{Experimental section}

\subsection{Materials}

3, 4-ethylenedioxythiophene (EDOT), tetrabutylammonium perchlorate, potassium 
ferricyanide $\left(\mathrm{K}_{3}\left[\mathrm{Fe}(\mathrm{CN})_{6}\right]\right)$, potassium ferrocyanide $\left(\mathrm{K}_{4}\left[\mathrm{Fe}(\mathrm{CN})_{6}\right]\right)$, hexaammineruthenium(III) chloride $\left(\left[\mathrm{Ru}\left(\mathrm{NH}_{3}\right)_{6}\right] \mathrm{Cl}_{3}\right)$, hexaammineruthenium(II) chloride $\left(\left[\mathrm{Ru}\left(\mathrm{NH}_{3}\right)_{6}\right] \mathrm{Cl}_{2}\right)$, toluidine blue $\mathrm{O}(\mathrm{TBO})$, potassium chloride $(\mathrm{KCl})$, tetrabutylammonium perchlorate (TBAP), anhydrous acetonitrile $\left(\mathrm{CH}_{3} \mathrm{CN}\right)$, ethanolamine $\left(\mathrm{EA}, \mathrm{NH}_{2} \mathrm{CH}_{2} \mathrm{CH}_{2} \mathrm{OH}\right)$, sodium L-lactate, N-(3dimethylaminopropyl)-N'-ethylcarbodiimide hydrochloride $\quad$ (EDC), Nhydroxysulfosuccinimide (S-NHS), fluorescein isothiocyanate conjugated bovine serum albumin (FITC-BSA), L-lactate dehydrogenase (LDH, from porcine heart, $250 \mathrm{U} \mathrm{mg}^{-1}$ ), ascorbic acid (AA) and uric acid (UA) were purchased from Sigma-Aldrich (USA). 3,4ethylenedioxythiophene derivative bearing 4-methoxy-4-oxobutanoic acid (EDOT-COOH, ${ }^{1} \mathrm{H}$ NMR spectrum in Figure S1) was provided by Shanghai Zhixing Chemical Technology (China). $\beta$-nicotinamide adenine dinucleotide reduced disodium salt hydrate (NADH) was bought from VWR (Sweden). Human serum (Type AB) was purchased from PAA Laboratories GmbH (Austria). $\beta$-Nicotinamide adenine dinucleotide solution (oxidised form, $\mathrm{NAD}^{+}$), disodium hydrogen phosphate monohydrate $\left(\mathrm{Na}_{2} \mathrm{HPO}_{4} \cdot \mathrm{H}_{2} \mathrm{O}\right)$ and potassium dihydrogen phosphate $\left(\mathrm{KH}_{2} \mathrm{PO}_{4}\right)$ were purchased from Merck (Darmstadt, Germany). $0.1 \mathrm{M}$ Phosphate buffer solutions (PBS 7.4) were prepared by mixing $\mathrm{Na}_{2} \mathrm{HPO}_{4}$ and $\mathrm{KH}_{2} \mathrm{PO}_{4}$ stock solution. All chemicals were of analytical grade and used without any further treatment. Deionised water from Milli-Q System was used throughout.

\subsection{Electropolymerisation of Nano-PEDOT-COOH}

Glassy-carbon electrodes (GCE, diameter $3 \mathrm{~mm}$; surface area $0.0707 \mathrm{~cm}^{2}$ ) were carefully polished with 0.3 and $0.05 \mu \mathrm{m}$ aluminum slurries and rinsed with deionised water under sonication. A mixture of monomer solution with a final concentration of $10 \mathrm{mM}$ was prepared by dissolving EDOT and EDOT-COOH monomers at different concentration in acetonitrile containing $0.1 \mathrm{M}$ TBAP. Different nanostructured PEDOT-COOH electrodes were electropolymerised in the $10 \mathrm{mM}$ monomer solution (containing 2.5, 5, 7.5, $10 \mathrm{mM}$ of EDOT$\mathrm{COOH})$ using a constant potential of $1.2 \mathrm{~V}\left(\mathrm{Ag} / \mathrm{Ag}^{+}\right.$as reference) with fixed charge of $20 \mathrm{mC}$. After rinsing with anhydrous acetonitrile, the resulting nanostructured PEDOT-COOH electrodes were dried in the air and denoted as Nano-PEDOT-COOH25\%, Nano-PEDOT- 
COOH50\%, Nano-PEDOT-COOH75\% and Nano-PEDOT-COOH100\%, respectively. Indium tin oxide coated glass (ITO) with a confined surface area of $1 \times 0.6 \mathrm{~cm}^{2}$ was used as an alternative working electrode during electropolymerisation for the characterisation experiments.

\subsection{Bio-conjugation of Nano-PEDOT-COOH}

The Nano-PEDOT-COOH interface was activated by applying $20 \mu \mathrm{L}$ of EDC (50 mM) and S-NHS $(50 \mathrm{mM})$ for 30 minutes followed by rinsing with water. $10 \mathrm{uL}$ of enzyme LDH (2 mg $\mathrm{mL}^{-1}$ ) was applied on the activated Nano-PEDOT-COOH surface and incubated for $2 \mathrm{~h}$. The unreacted carboxylic acid groups were then deactivated by applying $10 \mu \mathrm{L}$ of ethanolamine (1 $\mathrm{mM}$ ) for $30 \mathrm{~min}$. Finally, the LDH bio-conjugated Nano-PEDOT-COOH (Bio-Nano-PEDOT) electrodes were rinsed with PBS solution to remove excess enzymes. In order to examine the charge effect on the NADH response, ethanolamine was used to neutralise the carboxylic acid groups of the Nano-PEDOT-COOH to suppress the repulsion effect between the carboxylic acid groups and $\mathrm{NADH}$.

In parallel, fluorescence microscopy was used to characterise the bio-conjugation of NanoPEDOT-COOH using fluorescein isothiocyanate conjugated BSA (FITC-BSA). PEDOTCOOH50\%/ITO was treated with $100 \mathrm{uL}$ of EDC (50 mM) and sulfo-NHS (50 mM) for 30 minutes. After rinsing with water, $40 \mathrm{uL}$ of FITC-BSA $\left(1 \mathrm{mg} \mathrm{mL}^{-1}\right)$ was placed on the activated PEDOT-COOH surface for $2 \mathrm{~h}$. Excess FITC-BSA were then washed with water and dried under a gentle stream of nitrogen gas. As control, PEDOT-COOH50\%/ITO was treated with the same procedure with the exception of the activation of the carboxylic acid groups by EDC/SNHS.

\subsection{Characterisations and electrochemical measurements.}

Scanning electron microscopy (SEM) images were recorded using a LEO 155 Gemini (Zeiss, Germany) electron microscope. Fourier transform infrared (FTIR) spectroscopy was performed using a VERTEX spectrometer (Bruker, USA) equipped with an attenuated total reflection (ATR) measuring cell. The density of carboxylic acid groups on different NanoPEDOT-COOH biointerfaces were examined via toluidine blue O (TBO) staining assay (Meng et al. 2019a). In brief, PEDOT-COOH electrodes $\left(0.6 \mathrm{~cm}^{2}\right)$ were incubated in $2 \mathrm{mM}$ TBO 
solution $(0.1 \mathrm{mM} \mathrm{KOH})$ for $2 \mathrm{~h}$. Under alkaline conditions, the carboxylic acid groups deprotonate to bind with the positively charged TBO molecules with a ratio of $1: 1$. Nonspecifically bound TBO molecules were removed from the PEDOT-COOH surface by rinsing with $0.1 \mathrm{mM} \mathrm{KOH}$ solution several times. Finally, the samples were soaked in $5 \mathrm{~mL} 50 \%$ acetic acid for $30 \mathrm{~min}$ to desorb the TBO molecules. Absorbance spectroscopy was performed using a UV-vis spectrophotometer (UV-2450, Shimadzu, Japan) at $631 \mathrm{~nm}$ with an established standard calibration curve for TBO concentration vs. Absorbance (Regression equation: $\left.\mathrm{A}=0.0533+0.0589, \mathrm{R}^{2}=0.997\right)$.

All electrochemical measurements were performed using a CompactStat potentiostat (Ivium, Netherlands) at room temperature with a conventional three-electrode system comprising a platinum wire as the counter electrode, a glassy carbon working electrode, and a silver/silver chloride $(\mathrm{Ag} / \mathrm{AgCl})$ electrode as the reference. Electrochemical impedance spectroscopy (EIS) measurements were performed at PEDOT-COOH electrodes in $2 \mathrm{mM}$ $\mathrm{Fe}(\mathrm{CN}) 6^{3-/ 4-}$ in $0.1 \mathrm{M} \mathrm{KCl}$ over the frequency range $0.01 \mathrm{~Hz}$ to $100 \mathrm{kHz}$ and at $5 \mathrm{mV}$ amplitude. The impedance spectra were then fitted to an equivalent electrical circuit via ZSimpWin Software (AMETEK Scientific instruments).

\section{Results and discussion}

\subsection{Nanofibrous structure and carboxylic acid functionality of Nano-PEDOT-COOH}




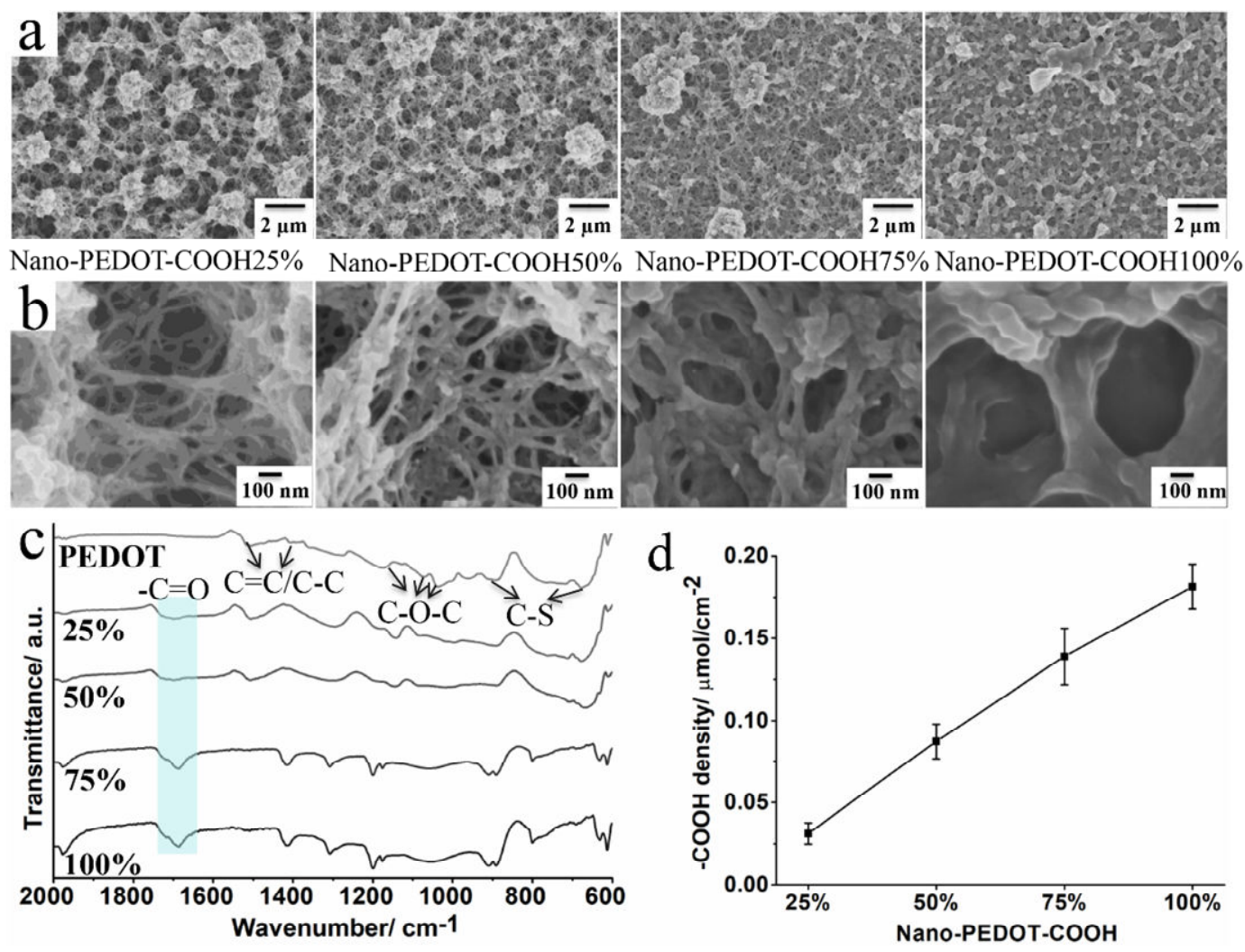

Figure 1. (a) SEM images of Nano-PEDOT-COOH with different amounts of EDOT-COOH ranging from 25-100\%; (b) high magnification SEM images; (c) FTIR spectra of PEDOT and nano-PEDOT-COOH25-100\%; (d) Carboxylic acid density on Nano-PEDOT-COOH25-100\% surface estimated by the TBO staining technique.

The surface morphology of various Nano-PEDOT-COOH films with different ratio of EDOT-COOH were examined using SEM (Figure 1a and 1b). Figure 1a shows that all NanoPEDOT-COOH films possess a network of interconnected nanofibres with varying fibre diameters supported by a spherical hub-like structure. With an increased amount of EDOT$\mathrm{COOH}$ (from $25 \%$ to $100 \%$ ), the aspect ratio of the resulting nanofibres decreased resulting in a relatively closed-structured film, which is ascribed to the hinderance due to the carboxylic acid side chains causing a steric effect. The high-magnification SEM images in Figure 1b show the nanofibrous morphology of the Nano-PEDOT-COOH25\%-100\%. The Nano-PEDOT$\mathrm{COOH} 25 \%$ and Nano-PEDOT-COOH50\% were composed of a three-dimensional network assembly of a relatively open-structured and thinner nanofibres with a fibre dimeter of $15.6 \pm$ $3.7 \mathrm{~nm}$ and $25.5 \pm 6.5 \mathrm{~nm}$, respectively. In contrast, the Nano-PEDOT-COOH75\% and Nano- 
PEDOT-COOH100\% were composed of relatively close-structured and thicker nanofibres with an increased fibre dimeter of $35.0 \pm 7.6 \mathrm{~nm}$ and $70.0 \pm 9.5 \mathrm{~nm}$, respectively. In addition to the nanofibrous structure, the presence of carboxylic acid groups was examined using ATR-FTIR (Figure 1c). The existence of bands at 1750 and $1680 \mathrm{~cm}^{-1}$ related to $-\mathrm{C}=\mathrm{O}$ for PEDOT$\mathrm{COOH} 25 \%-100 \%$, implies the successful co-polymerisation of EDOT-COOH into the NanoPEDOT-COOH matrix. The total surface density of carboxylic acid groups on the NanoPEDOT-COOH surfaces was quantified by the TBO staining technique as previously reported (Meng et al. 2019a; Rödiger et al. 2011). Figure 1d displays the linear increase of carboxylic acid group density from $0.03 \mu \mathrm{mol} \mathrm{cm} \mathrm{c}^{-2}$ for PEDOT-COOH25\% to $0.18 \mu \mathrm{mol} \mathrm{cm} \mathrm{cm}^{-2}$ for PEDOT$\mathrm{COOH} 100 \%$. These results reveal that the higher ratio of EDOT-COOH monomer contributes to higher carboxylic acid density on the resulting Nano-PEDOT-COOH surface and an increased diameter of the resulting nanofibrous structure.

\subsection{Effects of nanofibrous structure and carboxylic acid groups on Nano-PEPOT-COOH} electrochemical properties
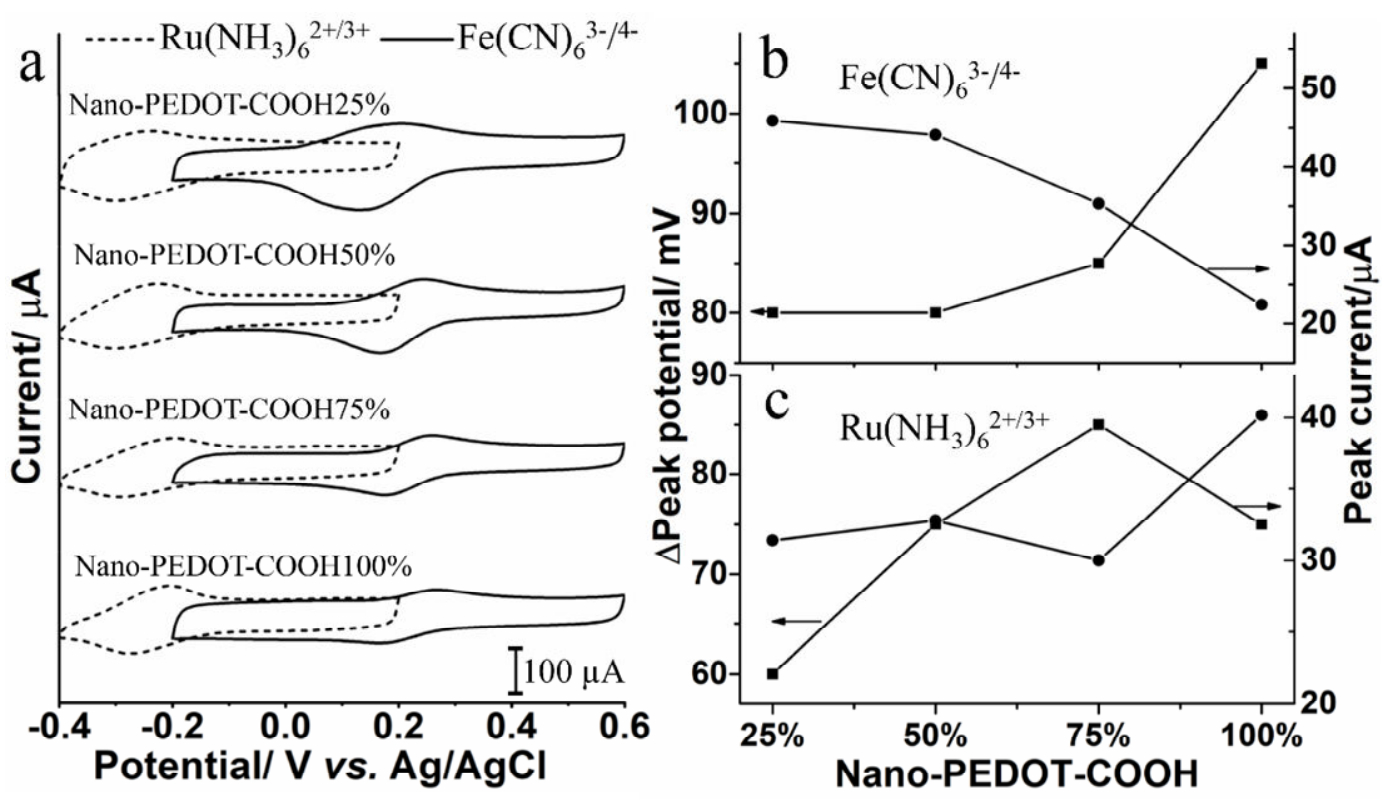

Figure 2. (a) Cyclic voltammograms of different Nano-PEDOT-COOH modified electrodes in $2 \mathrm{mM} \mathrm{Fe}(\mathrm{CN})_{6}^{3-/ 4-}$ (black curve), $\mathrm{Ru}\left(\mathrm{NH}_{3}\right)_{6}{ }^{2+/ 3+}$ (dashed curve) in $0.1 \mathrm{M} \mathrm{KCl}$; Corresponding redox peak potential and peak current of different Nano-PEDOT-COOH modified electrodes to 
$2 \mathrm{mM} \mathrm{Fe}(\mathrm{CN})_{6}^{3-/ 4-}$ (b) and $\mathrm{Ru}\left(\mathrm{NH}_{3}\right)_{6}{ }^{2+/ 3+}(\mathrm{c})$ in $0.1 \mathrm{M} \mathrm{KCl}$. Scan rate of $50 \mathrm{mV} \mathrm{s}^{-1}$.

The electrochemical properties of the series of Nano-PEDOT-COOH polymers with different nanostructure and carboxylic acid density were investigated by using two differently charged redox probes (i.e., negatively-charged $\mathrm{Fe}(\mathrm{CN})^{3-14-}$ and positively-charged $\left.\mathrm{Ru}\left(\mathrm{NH}_{3}\right)_{6}{ }^{2+/ 3+}\right)$. As shown in Figure 2a, all Nano-PEDOT-COOH modified electrodes exhibit quasi-reversible peaks with slightly different peak separations ( $\Delta \mathrm{Ep})$ and peak current (Ip) for both the redox probes. For the negatively-charged $\mathrm{Fe}(\mathrm{CN})_{6}^{3-/ 4-}$ probe (Figure $2 \mathrm{~b}$ ), the NanoPEDOT-COOH $25 \%$ showed the smallest $\triangle \mathrm{Ep}$ of $80 \mathrm{mV}$ and the highest anodic peak current of $45.9 \mu \mathrm{A}$, while a further increase the portion of EDOT-COOH in the Nano-PEDOT-COOH matrix resulted in increases of $\Delta \mathrm{Ep}$ and decreases of peak current, reaching a $\Delta \mathrm{Ep}$ of $105 \mathrm{mV}$ and an anodic peak current of $22.4 \mu \mathrm{A}$. These electrochemical characteristics can be ascribed to the successful incorporation of negatively-charged carboxylic acid groups on the NanoPEDOT-COOH interfaces repulsing the $\mathrm{Fe}(\mathrm{CN})_{6}^{3-/ 4-}$ probe, as well as the aspect ratio of the nanofibre structure (i.e. thicker and shorter length fibres) and relatively dense spatial arrangement limiting the accessible active surface of the nanofibres.

In contrast, the electrochemical characteristics of Nano-PEDOT-COOH towards positively-charged $\mathrm{Ru}\left(\mathrm{NH}_{3}\right)_{6}{ }^{2+/ 3+}$ can be seen in Figure 2c. In the range of Nano-PEDOT$\mathrm{COOH} 25-75 \%$, the nanostructure dominated the electrochemical properties; the NanoPEDOT-COOH interface composed of a nanofibrous structure with a higher aspect ratio (thinner and longer) showed a gradual reduction of $\Delta \mathrm{Ep}$ from $85 \mathrm{mV}$ to $60 \mathrm{mV}$ with improved electrode kinetics. Meanwhile, despite the fact that the Nano-PEDOT-COOH100\% was composed of nanofibres with a relatively lower aspect ratio, it showed a $\Delta \mathrm{Ep}$ of $75 \mathrm{mV}$; the electrostatic attraction of the positively-charged $\mathrm{Ru}\left(\mathrm{NH}_{3}\right)_{6}{ }^{2+/ 3+}$ by the relatively high carboxylic acid density compensated the effect of the nano-structure. At the same time, the anodic peak current of Nano-PEDOT-COOH25-75\% showed a relatively steady value of $\sim 30 \mu \mathrm{A}$, while the peak current of Nano-PEDOT-COOH100\% increased to $\sim 40 \mu \mathrm{A}$. 

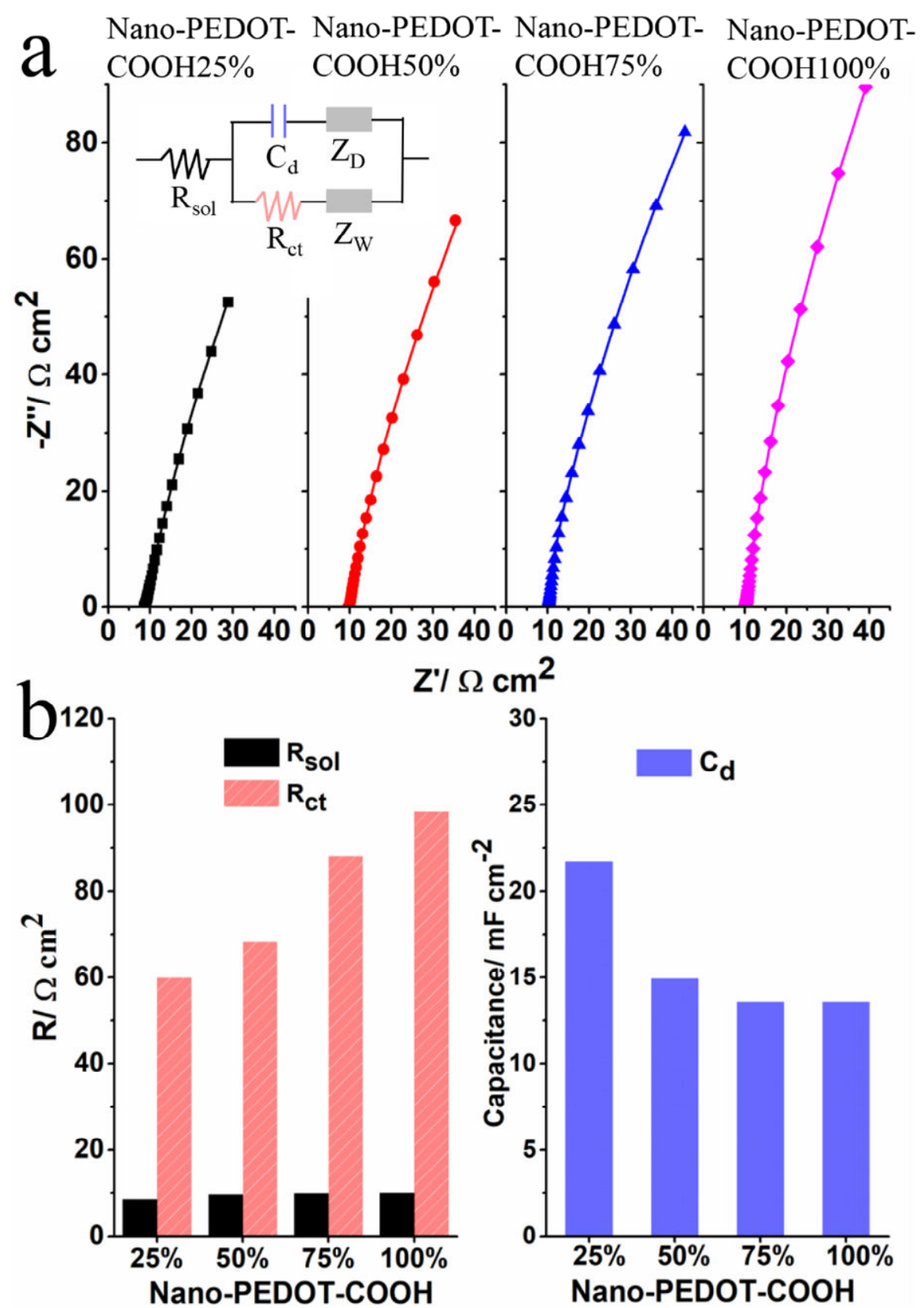

Figure 3. (a) EIS analysis of different Nano-PEDOT-COOH modified electrodes in Nyquist plots obtained in $2 \mathrm{mM} \mathrm{Fe}(\mathrm{CN})^{3-/ 4-}$ in $0.1 \mathrm{M} \mathrm{KCl}$; inset is the equivalent circuits; (b) corresponding curve fitting parameters.

Electrochemical impedance spectroscopy (EIS) was performed to evaluate the electrochemical properties for various Nano-PEDOT-COOH interfaces, as shown in Figure 3. An equivalent electrical circuit with adequate fit to the original data (inset of Figure 3a) was developed to model the behavior of Nano-PEDOT-COOH electrodes in $\mathrm{Fe}(\mathrm{CN})_{6}^{3-/ 4-}$ in $0.1 \mathrm{M}$ 
$\mathrm{KCl}$, comprising the solution resistance $\left(\mathrm{R}_{\mathrm{sol}}\right)$, the electron transfer resistance between the redox couple and the electrode interface $\left(R_{c t}\right)$, the electronic bulk capacitance $\left(C_{d}\right)$, the finite-length Warburg diffusion impedance $\left(Z_{D}\right)$ and the diffusion impedance of the redox probes in the solution $\left(Z_{W}\right)$ (Sundfors and Bobacka 2004; Sundfors et al. 2002). Figure 3b summarises the fitted parameters for various Nano-PEDOT-COOH interfaces. The $\mathrm{R}_{\text {sol }}$ for different NanoPEDOT-COOH interfaces remained similar. The $\mathrm{R}_{\mathrm{ct}}$ increased gradually as the carboxylic acid groups of the Nano-PEDOT-COOH increased, which is in good agreement with the trends in $\Delta E p$ and peak current obtained from the CVs shown in Figure $2 b$. The trend in $R_{c t}$ as a function of EDOT-COOH provides evidence of the successful incorporation of carboxylic acid groups into the Nano-PEDOT-COOH interfaces because of the increased electrostatic repulsion effect and relatively dense spatial interface with a higher EDOT-COOH component. Moreover, the $\mathrm{C}_{d}$ decreased as the carboxylic acid groups of the Nano-PEDOT-COOH increased, which is ascribed to the relatively dense spatial arrangement resulting in limited accessible active surface area at high amounts of EDOT-COOH.

\subsection{Effect on the nanofibrous structured PEDOT on NADH sensing}

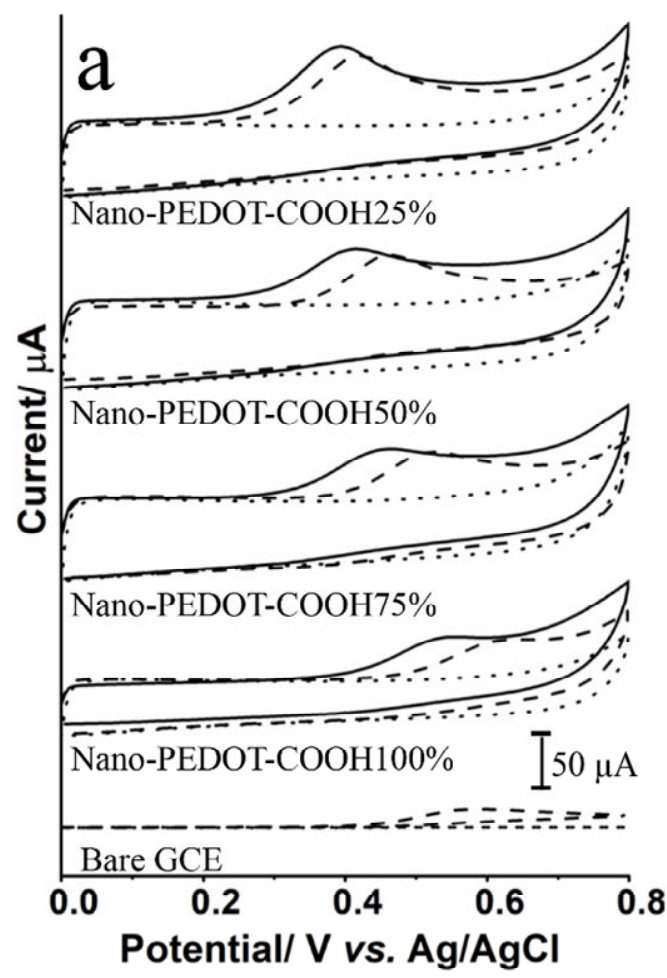

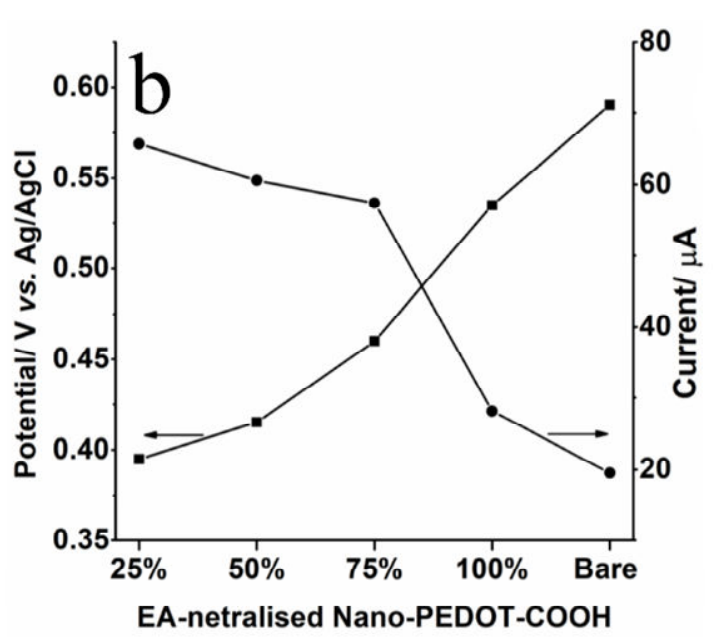


Figure 4. (a) CV responses of different Nano-PEDOT-COOH modified electrodes (dash: unneutralised, solid: EA-neutralised) with and without (dotted line) 2 mM NADH in 0.1 M PBS ( $\mathrm{pH}=7.4)$; (b) corresponding NADH oxidation peak potentials and peak currents of EAneutralised Nano-PEDOT-COOH modified electrodes to $2 \mathrm{mM} \mathrm{NADH}$ in $0.1 \mathrm{M}$ PBS (pH=7.4). Scan rate of $50 \mathrm{mV} \mathrm{s}^{-1}$.

The carboxylic acid moieties in the Nano-PEDOT-COOH primary polymer served as an intermediate functional group for bioconjugation that was reacted during covalent coupling of biomolecules or neutralised by the capping agent. Therefore, the electrochemical performance of the resulting Bio-Nano-PEDOT-based biosensor was mainly dominated by the net effect of the nano-structure morphology. NADH was chosen as the model to evaluate the biomolecules transducing property at the Nano-PEDOT-COOH interface, since NADH is one of the most important co-enzymes in the human body, and can be potentially coupled to a wide range of $\mathrm{NADH}$-dependent dehydrogenases for applications in biosensing and bioelectrocatalysis (Meng et al. 2018). Fig. 4a shows cyclic voltammograms of various Nano-PEDOT-COOH/GCE and bare GCE in the presence of $2 \mathrm{mM}$ NADH in 0.1 M PBS (pH=7.4). Compared to bare GCE, all Nano-PEDOT-COOH modified electrodes showed negatively shifted oxidation peak potentials and increased peak currents. In order to investigate the net effect of the nanostructured morphology towards NADH electrochemical oxidation, it was necessary to eliminate the electrostatic repulsion effect caused by the carboxylic acid groups. This was achieved by using ethanolamine (EA) to neutralise the carboxylic acid groups by conversion into hydroxy groups with EDC/S-NHS chemistry. Figure 4a shows the net effect on the nano-structured morphologies of various EA-neutralised Nano-PEDOT-COOH25-100\% electrodes with further negatively shifted oxidation peak potentials and increased peak currents. Figure $4 \mathrm{~b}$ summarises the key parameters from the CVs. We observed gradual decreases in peak potential from $0.53 \mathrm{~V}$ to $0.40 \mathrm{~V}$ and increases in peak current from $28.1 \mu \mathrm{A}$ to $68.5 \mu \mathrm{A}$, when the EAneutralised Nano-PEDOT-COOH was composed of a nanofibrous structure with a higher aspect ratio (i.e. Nano-PEDOT-COOH25\% has the highest aspect ratio nanofibrous structure, and 
Nano-PEDOT-COOH100\% has the lowest aspect ratio nanofibrous structure in Figure 1b). This result demonstrated that the nanofibrous structure with a high aspect ratio endowed the EAneutralised Nano-PEDOT-COOH interface with improved NADH sensing. In general, both the EA-neutralised Nano-PEDOT-COOH25\% and Nano-PEDOT-COOH50\% showed good electrochemical performance for detection of NADH (where Nano-PEDOT-COOH25\% was slightly better). However, for further biosensor development work, the key is to establish an effective signal transduction interface between the biomolecules and PEDOT. Therefore, it is necessary to compromise on the electrochemical performance with respect to the number of carboxylic acid functional groups for immobilisation of biomolecules. With this consideration, Nano-PEDOT-COOH50\% was chosen as a balance between a carboxylic acid group density of $0.08 \mu \mathrm{mol} \mathrm{cm} \mathrm{cm}^{-2}$ (Figure 1d) and good NADH oxidation properties with a relatively low peak potential of $0.42 \mathrm{~V}$ and a high peak current of $60.6 \mu \mathrm{A}$.

\subsection{NADH transduction and biomolecule conjugation for lactate biosensing}



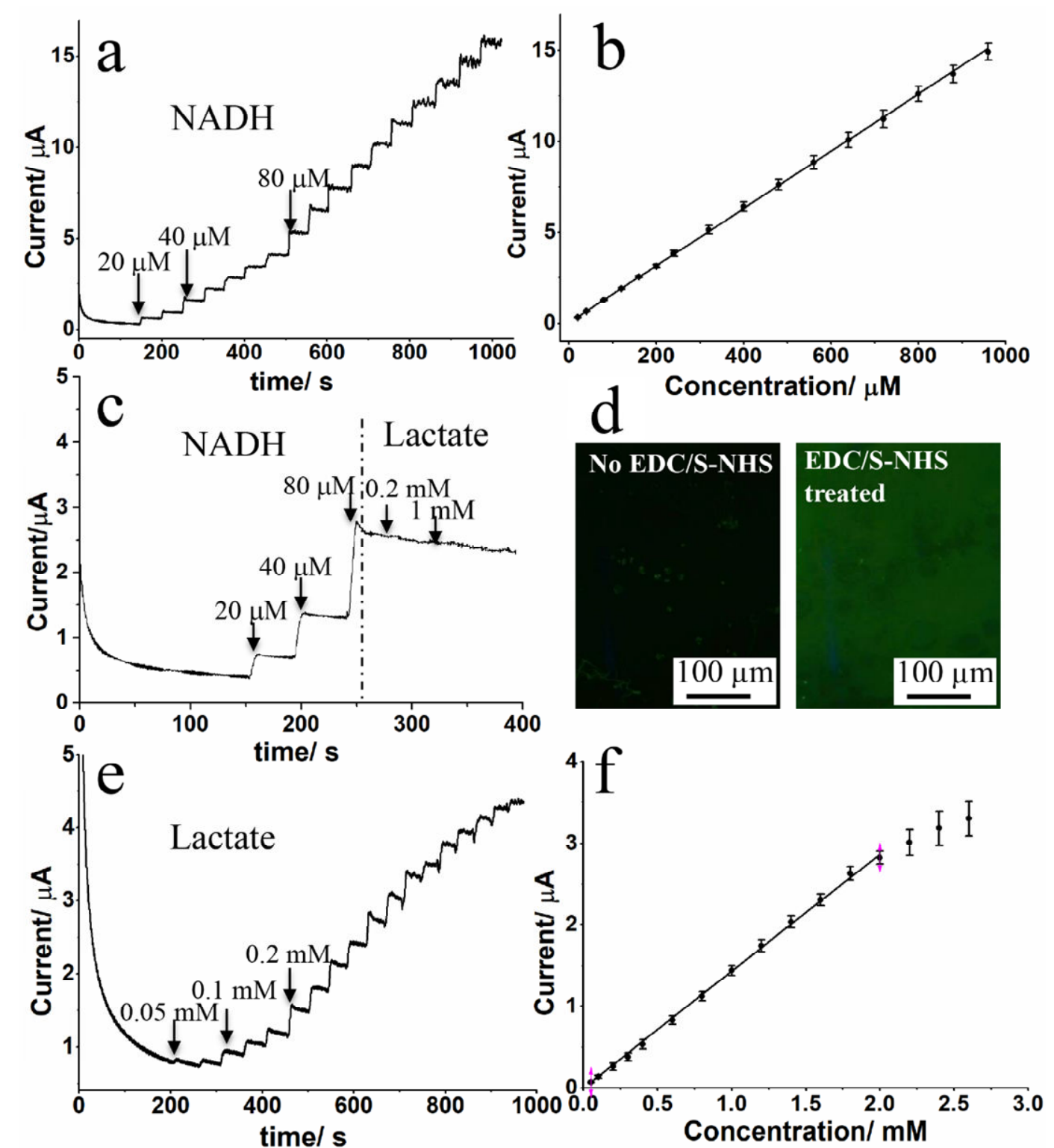

Figure 5. (a) Current-time response curve of EA-neutralised Nano-PEDOT-COOH50\% at $0.420 \mathrm{~V}$ for successive injections of $\mathrm{NADH}$ in $0.1 \mathrm{M}$ PBS (pH 7.4); (b) corresponding calibration curve for NADH sensing, $n=3$; (c) Current-time response curve of EA-neutralised Nano-PEDOT-COOH50\% at $0.420 \mathrm{~V}$ for varying concentration of NADH and lactate; (d) Fluorescence microscopy image of a FITC-BSA Bio-Nano-PEDOT interface without (left) and with (right) treatment; (e) Current-time response curve of LDH-Nano-PEDOT at $0.450 \mathrm{~V}$ for successive injection of lactate in 0.1 M PBS ( $\mathrm{pH} 7.4$ ); (f) corresponding calibration curve for lactate biosensing, $\mathrm{n}=3$.

The amperometric response of NADH on EA-neutralised Nano-PEDOT-COOH50\% was 
measured at $0.42 \mathrm{~V}$ by successive addition of NADH in $0.1 \mathrm{M}$ PBS (pH 7.4). As can be seen from Figure 5a, EA-neutralised Nano-PEDOT-COOH50\% modified electrodes exhibited a fast response to the successive additions of NADH and reached a $95 \%$ steady-state current within 5 s. The corresponding calibration curves are shown in Figure $4 \mathrm{~b}$. The response current of EAneutralised Nano-PEDOT-COOH50\% modified electrode was linearly proportional to NADH concentration over a wide linear range of $20-960 \mu \mathrm{M}\left(\mathrm{R}^{2}=0.997\right)$. The sensitivity for NADH sensing was calculated to be $0.224 \mu \mathrm{A} \mu \mathrm{M}^{-1} \mathrm{~cm}^{-2}$, with a detection limit of $2.04 \mu \mathrm{M}$ ( $3 \sigma /$ slope). The analytical performance was compared with other conventional carbon-based (graphene, CNTs), polymer-based electrodes and their composites with metal nanoparticles described in the literature (Table S1). It is important to note that those reports are mainly based on nanocatalyst modified electrodes such as graphene, CNT, metal nanoparticles and advanced polymer composite doped with nano-catalysts, while our 3D nanofibrous PEDOT network without nanocatalyst could detect NADH at a relatively low oxidation peak potential with comparable sensitivity and wide linear range, due to its high surface area and porous structure to facilitate signal transduction. Moreover, PEDOT possesses several inherent advantages such as light weight, flexibility, ease of fabrication, low cost and good biocompatibility as a sensing interface.

A Bio-Nano-PEDOT-based biosensor was constructed using NADH-dependent lactate dehydrogenase (LDH) coupled with the Nano-PEDOT-COOH50\%. The control experiment (Figure 5c) displayed no observable response to successive injections of 0.2 and $1 \mathrm{mM}$ lactate with the Nano-PEDOT-COOH electrode. In order to visualise the successful conjugation of biomolecules onto the Nano-PEDOT-COOH50\% interface, a fluorescently-labelled FITC-BSA protein was used as a model that covalently bound onto the Nano-PEDOT-COOH50\% via EDC/S-NHS chemistry and then the interface was examined using fluorescence microscopy. Figure 5d shows the fluorescence micrograph of the resulting FITC-BSA conjugated NanoPEDOT-COOH50\% with a strong, well-covered and homogenous distribution of the fluorescence signal, which indicates a good immobilisation efficiency, while the control experiment without EDC/S-NHS activation of carboxylic acid groups showed a weak and insignificant fluorescence signal, indicating that the immobilisation was not due to non-specific 
physical adsorption onto the Nano-PEDOT interface.

After bioconjugation of $\mathrm{LDH}$, the analytical performance of the LDH-Nano-PEDOT biosensor for lactate detection was evaluated. Figure 5e shows typical current-time curve of the LDH-Nano-PEDOT biosensor responses to successive addition of lactate at $0.45 \mathrm{~V}$ lactate in 0.1 M PBS ( $\mathrm{pH} 7.4$ ) containing $3 \mathrm{mM} \mathrm{NAD}^{+}$. A stepwise increase in current response was observed for each successive addition of lactate, reaching $95 \%$ of the steady-state current within 10 s. The steady state current response (Figure 5f) was linear with respect to lactate concentration over the range of $0.05-1.8 \mathrm{mM}\left(\mathrm{R}^{2}=0.995\right)$ with a sensitivity of $20.26 \mu \mathrm{A} \mathrm{mM}^{-1}$ $\mathrm{cm}^{-2}$. The biosensing mechanism is based on a relay bioelectrochemical signal transduction between the LDH (bioorganic molecule) and the Nano-PEDOT (organic molecule), during which LDH catalyses the conversion of lactate to pyruvate and NADH in the presence of $\mathrm{NAD}^{+}$, followed by the Nano-PEDOT electrode catalytically oxidising NADH to generate a signal current. The linear range of the all-polymer lactate biosensor was comparable, or in some cases better, than reported values of other advanced nanomaterial-based biosensors in the literature, such as $\mathrm{Fe}_{3} \mathrm{O}_{4} / \mathrm{MWCNTs}(\sim 300 \mu \mathrm{M})$ (Teymourian et al. 2012), pTTCA/MWNT (5-90 $\mu \mathrm{M})($ Rahman et al. 2009), Metal nitride/Graphene Nanohybrids (10-220 $\mu \mathrm{M})$ (Wen et al. 2011) and $\mathrm{Au}$ NPs silicate network (100-600 $\mu \mathrm{M})$ (Jena and Raj 2006). The selectivity, storagestability and practical applicability of the fabricated lactate biosensor in serum samples were examined and the results are shown in Figure S2. The selectively of the biosensor was evaluated against the common interfering electroactive species uric acid and ascorbic acid, which showed insignificant responses. The stability of the biosensor was tested for 7 days storage in PBS at $4{ }^{\circ} \mathrm{C}$, and it retained $95 \%$ of its signal responses after storage for 5 days, while the signal responses decreased to $78 \%$ at 7 days, likely due to enzyme degradation. We demonstrated the practical use of the biosensor for detection of various concentration of lactate in spiked serum samples and obtained a high recovery value of (95.8-101\%). Thus, we successfully demonstrated a Bio-Nano-PEDOT (all-bioorganic/organic polymer) biosensor that did not require metal/carbon nanocatalysts by combining functionalisation and nanostructure formation strategies, thus providing a sound basis for future development of a range of advanced NADH- 
dehydrogenase coupled all-polymer-based wearable and flexible biosensors.

\section{Conclusions}

We have demonstrated a facile approach for simultaneous preparation of a Nano-PEDOT$\mathrm{COOH}$ interface with a tunable 3D nanofibrous network and carboxylic acid groups via controlled co-polymerisation of EDOT and EDOT-COOH monomers at different ratios using tetrabutylammonium perchlorate as a soft-template. The nanofibres assembled into a threedimensional porous network with high specific surface area, which resulted in a low charge transfer resistance for probes used with CV and EIS, and excellent oxidation of the co-enzyme NADH over a wide linear range and with high sensitivity. The carboxylic acid groups on the Nano-PEDOT-COOH (organic molecule) provided anchoring sites for the stable immobilisation of NADH-dependent lactate dehydrogenase (bioorganic molecule) via EDC/SNHS chemistry for the fabrication of Bio-Nano-PEDOT, thus realising an all-polymer biosensor without the use of traditional nanocatalysts such as carbon materials, metal, metal oxides or dyes. This Bio-Nano-PEDOT interface provides a sound basis for future development of a wide range of advanced NADH-dehydrogenase coupled biosensors fabricated entirely from polymers. This paves the way for the creation of new bioorganic electronic devices for sensing, biotransformation and energy generation.

\section{Acknowledgments}

The authors would like to acknowledge the Swedish Research Council (VR-2015-04434) and the China Scholarship Council (File no. 201606910036) for generous financial support to carry out this research.

\section{References}

Goda, T., Toya, M., Matsumoto, A., Miyahara, Y., 2015. ACS Appl. Mater. Interfaces 7(49), 27440-27448.

Godeau, G., Darmanin, T., Guittard, F., 2016. J. Appl. Polym. Sci. 133(11).

Hai, W., Goda, T., Takeuchi, H., Yamaoka, S., Horiguchi, Y., Matsumoto, A., Miyahara, Y., 2017. ACS Appl. Mater. Interfaces 9(16), 14162-14170. 
Inal, S., Rivnay, J., Suiu, A.-O., Malliaras, G.G., McCulloch, I., 2018. Acc. Chem. Res. 51(6), 1368-1376.

Jena, B.K., Raj, C.R., 2006. Anal. Chem. 78(18), 6332-6339.

Jin, H., Guo, H., Gao, X., Gui, R., 2018a. Sens. Actuator B-Chem. 277, 14-21.

Jin, H., Zhao, C., Gui, R., Gao, X., Wang, Z., 2018b. Anal. Chim. Acta 1025, 154-162.

Lin, K.-C., Tsai, T.-H., Chen, S.-M., 2010. Biosens. Bioelectron. 26(2), 608-614.

Lin, Y.-F., Li, C.-T., Ho, K.-C., 2016. J. Mater. Chem. A 4(2), 384-394.

Lu, C.-F., Liao, S.-F., Wang, K.-H., Chen, C.-T., Chao, C.-Y., Su, W.-F., 2019. Nanoscale 11(43), 20977-20986.

Luo, S.-C., Xie, H., Chen, N., Yu, H.-h., 2009. ACS Appl. Mater. Interfaces 1(7), 1414-1419.

Mantione, D., del Agua, I., Schaafsma, W., Diez - Garcia, J., Castro, B., Sardon, H., Mecerreyes, D., 2016. Macromol. Biosci. 16(8), 1227-1238.

Meng, L., Turner, A.P., Mak, W.C., 2018. Biosens. Bioelectron. 120, 115-121.

Meng, L., Turner, A.P., Mak, W.C., 2019a. ACS Appl. Mater. Interfaces 11(37), 34497-34506.

Meng, L., Turner, A.P., Mak, W.C., 2019b. Biotechnol. Adv. 39, 107398.

Nguyen, V.-Q., Schaming, D., Martin, P., Lacroix, J.-C., 2015. ACS Appl. Mater. Interfaces 7(39), 21673-21681.

Rahman, M., Shiddiky, M.J., Rahman, M.A., Shim, Y.-B., 2009. Anal. Biochem. 384(1), 159165.

Rödiger, S., Ruhland, M., Schmidt, C., Schröder, C., Grossmann, K., Böhm, A., Nitschke, J.r., Berger, I., Schimke, I., Schierack, P., 2011. Anal. Chem. 83(9), 3379-3385.

Sekine, J., Luo, S.C., Wang, S., Zhu, B., Tseng, H.R., Yu, H., 2011. Adv. Mater. 23(41), 47884792.

Sundfors, F., Bobacka, J., 2004. J. Electroanal. Chem. 572(2), 309-316.

Sundfors, F., Bobacka, J., Ivaska, A., Lewenstam, A., 2002. Electrochim. Acta 47(13-14), 22452251 .

Teymourian, H., Salimi, A., Hallaj, R., 2012. Biosens. Bioelectron. 33(1), 60-68.

Travas-Sejdic, J., Aydemir, N., Kannan, B., Williams, D., Malmström, J., 2014. J. Mater. Chem. B 2(29), 4593-4609.

Wang, G., Morrin, A., Li, M., Liu, N., Luo, X., 2018. J. Mater. Chem. B 6(25), 4173-4190.

Wen, Z., Cui, S., Pu, H., Mao, S., Yu, K., Feng, X., Chen, J., 2011. Adv. Mater. 23(45), 54455450 . 
Tunable 3D nanofibrous and bio-functionalised PEDOT network explored as a conducting polymer-based biosensor

Meng, Lingyin

Elsevier

Meng L, Turner APF, Cheung-Mak W. (2020) Tunable 3D nanofibrous and bio-functionalised PEDOT network explored as a conducting polymer-based biosensor. Biosensors and Bioelectronics, Volume 159, July 2020, Article number 112181

https://doi.org/10.1016/j.bios.2020.112181

Downloaded from Cranfield Library Services E-Repository 